\title{
Dielectric and Conductivity Properties of some Wood Composites
}

\author{
K.Ch.Varada Rajulu*, B.N Mohanty \\ Indian Plywood Industries Resarch and Training Institute \\ IPIRTI, P. B. No 2273, Tumkur road, Bangalore, 60022 \\ *varadaraju6@gmail.com, Mobile: +918904433282, Fax: +91-80-2839-6361
}

\section{Keywords: Dielectric, Temperature, Frequency, Conductivity}

\begin{abstract}
This study presents the dielectric and conductivity properties as function of temperature and frequency of wood based composites. These properties were measured by an open-ended coaxial probe at frequency range between $100 \mathrm{kHz}$ to $100 \mathrm{MHz}$, temperature from $30^{\circ} \mathrm{C}$ to $200^{\mathrm{O}} \mathrm{C}$ which is fully computer interfaced. It has been observed that dielectric constant $\left(\varepsilon^{\prime}\right)$ and dielectric loss factor $\left(\varepsilon^{\prime \prime}\right)$ increase with increasing temperature and decrease with increasing frequency. At low temperature region, the conductivity depends significantly on the frequency. However, with the increase in temperature dielectric relaxation takes place and the dependency of the conductivity on frequency get reduced. The patterns of variation were established for the studied specimens and discrepancies were discussed. The study of dielectric properties will help in improving the drying, heating and gluing processes of wood and wood based products.
\end{abstract}

\section{INTRODUCTION}

The dielectric properties of wood are useful for the understanding of molecular structure of wood, instrumental and industrial applications. Recently many researchers are exploring the utilization of high frequency and microwave techniques for improving energy efficiency and quality of end product. The dielectric properties of wood have been studied in the past by using several techniques.

When dielectric (wood or wood composite) material in an electric field, the current-carrying properties of wood/wood composites are governed by certain properties such as moisture content, density, grain direction, temperature etc. The overall effects of these parameters interact with each other and add to the complexities of the dielectric properties. The temperature dependence of wood dielectric properties have been reported by many researchers by using different methods [James w.l. et al, James w.l.et al, D.W. Hamil et al] [1-3].

The dielectric properties of a non-conducting material describe the interaction of the material with electric fields. The two interactions of primary interest are the absorption and storage of electric potential energy in the form of polarization within the dielectric material, and the dissipation or loss of part of this energy when dielectric field is removed. The energy absorbed by dielectric is most easily measured in terms of capacitance, so the dielectric constant of material is usually defined as the ratio of capacitance with the material as the insulating medium to the capacitance of the same invacuum. The rate of energy loss in the dielectric is expressed commonly by the loss factor (or dissipation factor) dissipation factor is the product of loss tangent and dielectric constant.

One of the earlier studies of the dielectric properties of wood was studied by C. Skaar et al[4], who showed that the dielectric constant of wood increased continuously as the moisture content increased and decreased with increasing frequency of the applied field. His data do not show clearly recognizable trends in loss tangent. Dielectric properties at low frequencies and low moisture content have been reported by several researchers W.L. James et al, J. Rafalski et al [1,5] it has been established that the dielectric behaviour of wood to an alternating electric field are governed by several factors such as frequency, temperature, moisture content, density and grain direction R.T. Lin et al, G.I. Torgovnikov et al [6,7]. The dielectric properties of were carried out for typical Australian wood based on Von Hippel's transmission line method in microwave frequency region by Ramaswamy et al [8]. As the studies of dielectric properties became more 
sophisticated, other variables were considered such as temperature, Structural direction M Norimoto et al [9] and density J.Rafalski et al[5] with the results showing that these other variables also have important influence on the dielectric behaviour of wood. A notable weakness in the literature of dielectric and electrical properties of wood based composite materials have been lack of study that covered a wide range of all primary variables of sample completely.

In this present study the dielectric constant $\left(\varepsilon^{\prime}\right)$, dielectric loss factor $\left(\varepsilon^{\prime \prime}\right)$, conductivity of some of wood based composite materials such as Fire Retardant Plywood (FR Ply), Moisture Resistant Plywood (MR Ply), Boiling Water Proof Plywood (BWP Ply), and Medium Density Fibre board (MDF). Data were taken at temperature from $30^{\circ} \mathrm{C}$ to $200^{\circ} \mathrm{C}$ and frequency range from $100 \mathrm{kHz}$ to $100 \mathrm{MHz}$.

\section{MATERIALS AND METHODS}

\section{Specimen:}

The specimen from each board were milled and cut into disc with $30 \mathrm{~mm}$ diameter and $5 \mathrm{~mm}$ thickness for testing. Two set of samples were prepared for each material. One specimen used as a control to calculate the initial moisture content and second specimen for the data measurements. The samples were placed in a conditioning chamber at recommended temperature $\left(27 \pm 2{ }^{\mathrm{O}} \mathrm{C}\right)$ and humidity $(65 \pm 5 \%)$ to achieve recommended moisture content 5-15\%. After samples were conditioned, moisture content was calculated. The average moisture content variation was $\pm 3 \%$.

In order to obtain reproducible data, it is necessary to eliminate variability in the electrochemical condition of the specimen surfaces. This was achieved by coating the flat surfaces (both) of the specimens with electrically conducting silver paste. After electrode specimen were dried at $50^{\circ} \mathrm{C}$ for $30 \mathrm{mins}$.

Dielectric and conductivity properties of some wood composite materials have been studied those are Fire Retardant Plywood (FR Ply), Moisture Resistant Plywood (MR Ply), Boiling Water Proof Plywood (BWP Ply), and Medium Density Fibre board (MDF). Data were taken at temperature from $30^{\circ} \mathrm{C}$ to $200^{\circ} \mathrm{C}$ and frequency range from $100 \mathrm{kHz}$ to $100 \mathrm{MHz}$.

\section{Testing:}

Dielectric constant, loss tangent and conductivity data were taken by using HIOKI make LCR Hi-Tester, Japan with frequency range of $100 \mathrm{kHz}$ to $120 \mathrm{MHz}$. The system consisting of an open-ended coaxial probe ( $4 \mathrm{~mm}$ diameter) and fully computer interfaced. The system measured the reflection coefficient data from open and short. For temperature variation hot air circulated oven range from Room temperature (RT) to $600^{\circ} \mathrm{C}$ attached to the system.

\section{Accuracy and Errors}

The instruments used in this study were capable of measuring the capacitance and dissipation factor of stable capacitors to high degree of precision and accuracy, so the predominant source of error here was the inherent instability of the quantities being measured. Data taken on specimens encapsulated as described earlier were generally constant and repeatable to within $\pm 10 \%$ of the mean data.

\section{RESULTS AND DISCUSSION}

Each specimen was placed in sample holder and measurements were taken at different temperatures. The temperature was raised using a PID controlled heating system with an accuracy of $\pm 0.1^{\mathrm{O}} \mathrm{C}$. Before measurement at each temperature, the specimen was kept at this constant temperature for $5 \mathrm{~min}$ to ensure that the sample stabilized at a uniform internal temperature and equilibrated with the surrounding cell temperature.

Dielectrics are the substances, which do not possess free electric charges under ordinary circumstances. In fact the most important property of dielectrics is their ability to become polarized under the action of an external electric field. An external electric field influences the atoms and 
molecules of dielectrics and hence the positive charged particles are pushed in the direction of the electric field, while the negative charged particles are pushed in the opposite direction from their equilibrium position. Hence dipoles are developed and they produce a field of their own. The process of producing electric dipoles out of neutral atoms and molecules is referred to as polarization.

\section{Dielectric constant and dielectric loss factor:}

Dielectric constant $\left(\varepsilon^{\prime}\right)$ was calculated by using the following relation

$$
\varepsilon^{\prime}=\mathrm{C} / \mathrm{C}_{\mathrm{o}}
$$

where, $\mathrm{C}$ and $\mathrm{C}_{\mathrm{o}}$ are the capacitance values with and without dielectric respectively. $\mathrm{C}_{\mathrm{o}}$ is given by

$$
\mathrm{C}_{\mathrm{o}}=[(0.08854 \mathrm{~A}) / \mathrm{d}]
$$

where, A is the area of the electrode surface of the specimen and d thickness of the specimen. Dielectric loss tangent (Tan $\delta$ ) is defined as follows

$$
\operatorname{Tan} \delta=\varepsilon^{\prime \prime} / \varepsilon^{\prime}
$$

where, $\varepsilon^{\prime \prime}$ is the dielectric dissipation factor.

Dielectric constant and loss factor of wood based composite materials have been measured by using LCR meter with temperature range from $30^{\circ} \mathrm{C}$ to $200^{\circ} \mathrm{C}$ with $10^{\circ} \mathrm{C}$ intervals and frequency from $100 \mathrm{kHz}$ to $100 \mathrm{MHz}$. Fig.1 shows the variation of dielectric constant $\left(\varepsilon^{\prime}\right)$ with temperature for all the tested specimens. The dielectric constant is increases with increasing in temperature. The increase in di electric constant with temperature can be explained by the fact that the dipolar groups are bound in the solid structures so that the dipole is a structural element of solid lattice and the rigidity of the lattice hinders (delay) the orientation of the dipoles A.Z. Nanassy et al [10]. At elevated temperatures, the dipoles acquire energy, which allows them to reorient, consequently an increase in the dielectric constant results. These results support the finding of J. Tsutsumi et al[11], who showed the dielectric constant of wood increased with temperature and also showed as strong relationship with frequency. Fig. 2 shows the behaviour of dielectric loss factor $\left(\varepsilon^{\prime \prime}\right)$ with temperature at different frequencies. The values are increased with increasing temperature due to orientation, space charge and interfacial polarization contributions.

Fig. 3 shows the behaviour of real part of dielectric constant $\left(\varepsilon^{\prime}\right)$ verses frequency response at different temperatures. It is observed that $\varepsilon^{\prime}$ follows an inverse dependence on frequency, normally followed by almost all dielectric/ferroelectric materials. The higher values of dielectric constant found at low frequency is due to the presence of all types of polarization (atomic, electronic, space charge etc.) mechanisms Mike Golio et al [12]. At higher frequencies $\varepsilon^{\prime}$ are found to decrease, as it is arise due to the contribution from electronic polarization, other contributions becoming insignificant and also fall in dielectric constant arises from the fact that polarization does not occur instantaneously with the application of the electric field because of inertia Mike Golio et al [12]. This takes place at the contacts or in the bulk of the material at the grain boundary. Charge is able to accumulate at these interfacial sites and thereby able to contribute to the total capacity or dielectric constant. At low frequencies, all types of polarization contribute. As frequency is increased, those with large relaxation times cease to respond and hence the decrease in dielectric constant. Dielectric and conductivity values of all the studied composite materials are shown in table 1.

\section{Conductivity:}

The mechanism of ionic conductivity in solids has been generally discussed in terms of the conventional hopping formalism A.B. Lidiard [13]. The conductivity of an ionic conductor is determined by both the concentration of the mobile ions and the rate at which they are able to hop from site to site in the material. Fig. 4 shows the conductivity of tested specimens as a function of 
frequency $(100 \mathrm{kHz}$ to $100 \mathrm{MHz})$ at different temperatures. The characteristic features of these dielectric materials are

(i) A Plateau region at low frequencies which reflects the d.c. conductivity of the samples and

(ii) A dispersive region at high frequencies where the conductivity can be described by a power law.

$$
\sigma(\omega) \propto \omega^{n}
$$

where $\omega$ is the frequency and $n$ is the exponential factor, which generally varies between 0 and 1 . Both regions are separated by a cross-over regime. The conductivity in the low frequency region is associated with the short range translational hopping mechanism and conductivity in the high frequency region is associated with the reorientational hopping mechanism. This over all behaviour has been found for many ionic conductors and has been reported by many researchers K.Funke et al [14], W.Dieterich et al [15].

Temperature dependence of conductivity at different frequencies of studied specimens is shown in Fig.5. It was found that conductivity increased with increase in temperature. Orientation of fiber's in composite changes the structure of the total composite, due to which conductivity changes with the orientation. At low temperature region, the conductivity depends significantly on the frequency. However, with the increase in temperature dielectric relaxation takes place and the dependency of the conductivity on frequency get reduced.

\section{CONCLUSIONS}

Dielectric constant and loss tangent of wood based composite materials have been measured by using LCR meter with temperature range from $30^{\circ} \mathrm{C}$ to $200^{\circ} \mathrm{C}$ with $10^{\circ} \mathrm{C}$ intervals and frequency from $100 \mathrm{kHz}$ to $100 \mathrm{MHz}$. Higher values of dielectric constant $\left(\varepsilon^{\prime}\right)$ and dielectric loss factor $\left(\varepsilon^{\prime \prime}\right)$ found at low frequency is due to the presence of all types of polarization (atomic, electronic, space charge etc.) mechanisms. Observed conductivity is found to dependent on both temperature and frequency indicating the conduction mechanism is thermally activated process in all the studied materials. The results obtained using the coaxial probe appeared to be reasonable when compared with previous studies. This study help in improving energy efficiency and quality of end product are exploring the utilization of high frequency and microwave techniques.

\section{Acknowledgement}

The author would like to sincerely thanks to Director, IPIRTI, Bangalore for permission and for providing necessary research facilities.

\section{Tables:}

Table 1: Dielectric and Conductivity values of wood and bamboo based composite materials

\begin{tabular}{|c|c|c|c|c|c|}
\hline S1.No & Specimen name & \multirow{2}{*}{$\begin{array}{c}\text { Moisture } \\
\text { content at }\end{array}$} & \multicolumn{2}{|c|}{$\begin{array}{c}\text { Dielectric constant } \\
\text { (at 100 kHz) }\end{array}$} & \multirow{2}{*}{\begin{tabular}{c} 
Conductivity at \\
RT \\
\cline { 4 - 5 }
\end{tabular}} \\
\cline { 4 - 5 } & & $\begin{array}{c}\text { RT (before } \\
\text { test), } \%\end{array}$ & low & Maximum & \\
\hline 1 & MDF & 7 & 0.94 & 1.31 & $1.029 \times 10^{-7}$ \\
\hline 2 & MR plywood & 6 & 6.62 & 7.27 & $9.76 \times 10^{-8}$ \\
\hline 3 & BWP plywood & 6 & 6.68 & 7.25 & $1.01 \times 10^{-7}$ \\
\hline 4 & FR plywood & 6 & 7.12 & 8.63 & $9.76 \times 10^{-8}$ \\
\hline
\end{tabular}


Figures:

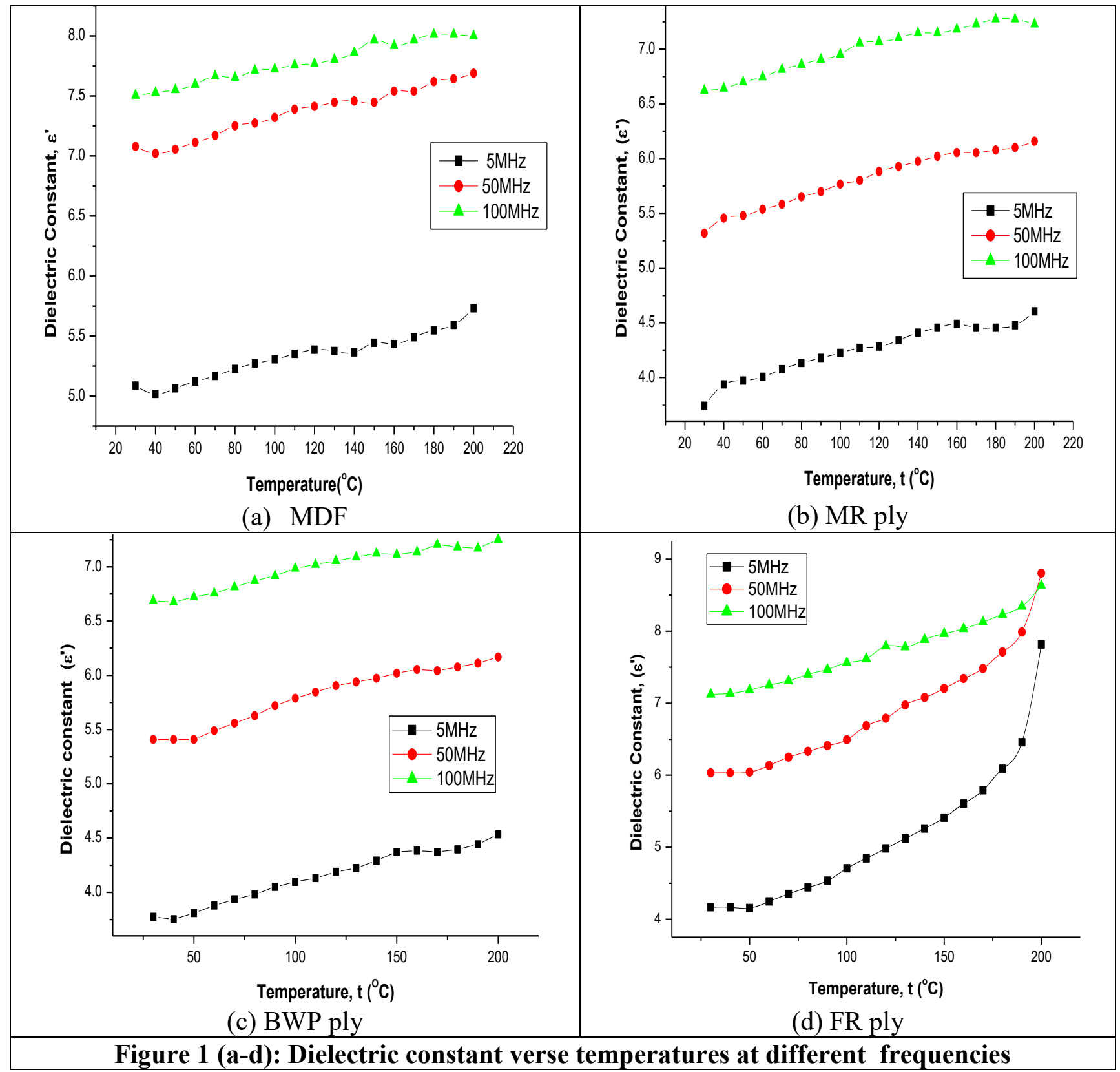



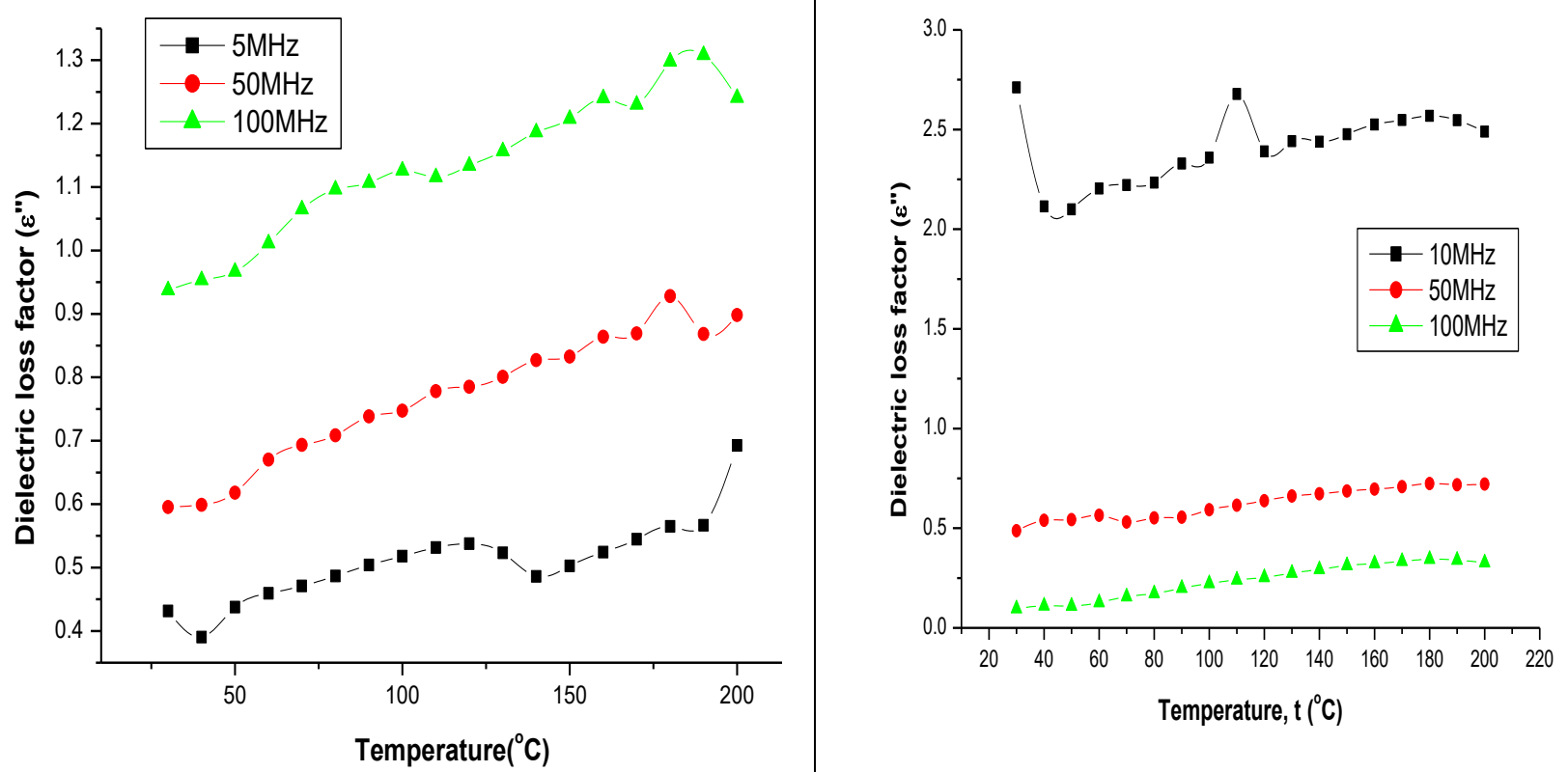

(a) MDF

(b) MR ply
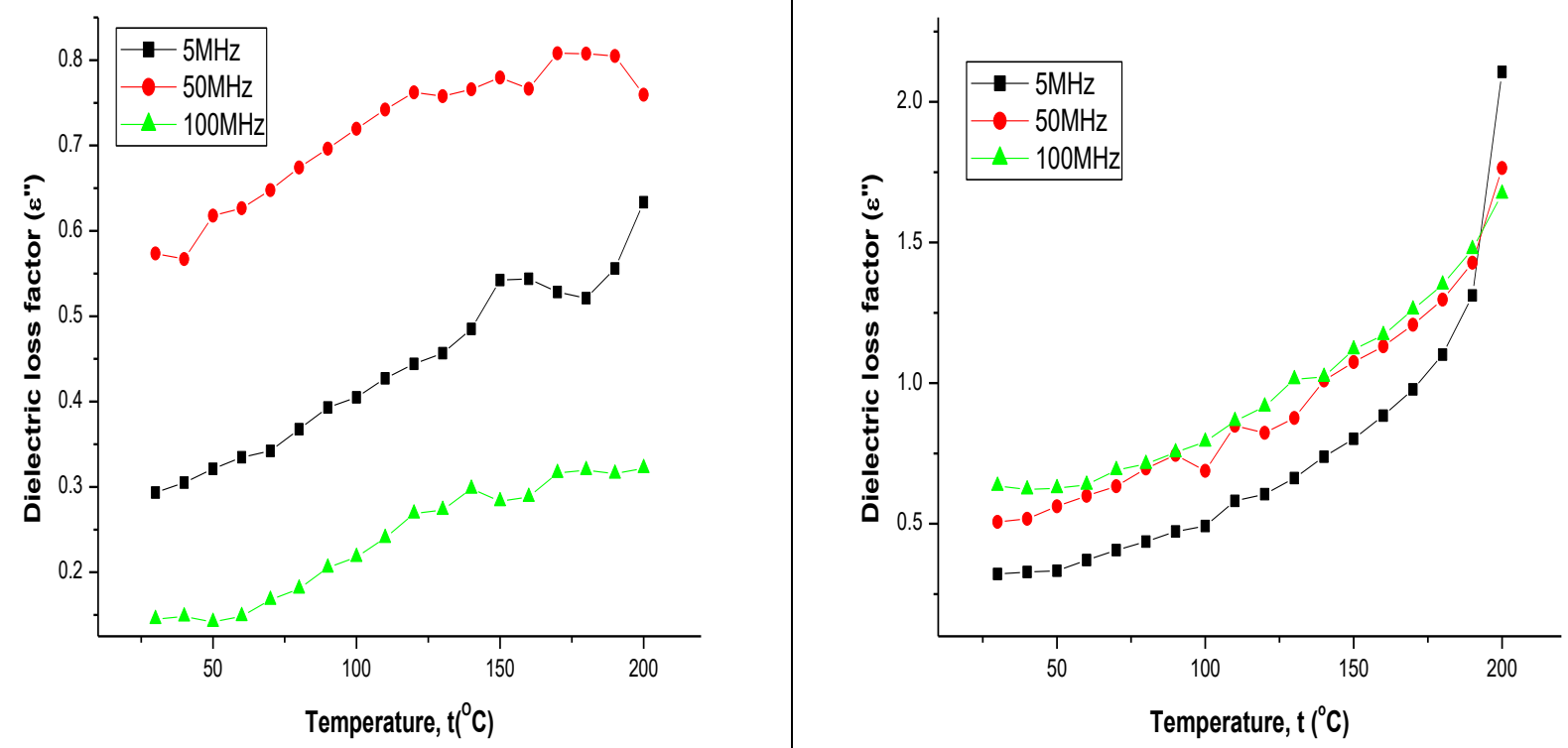

(c) BWP ply

(d) FR ply

Figure 2(a-d): Dielectric constant verse temperatures at different frequencies 


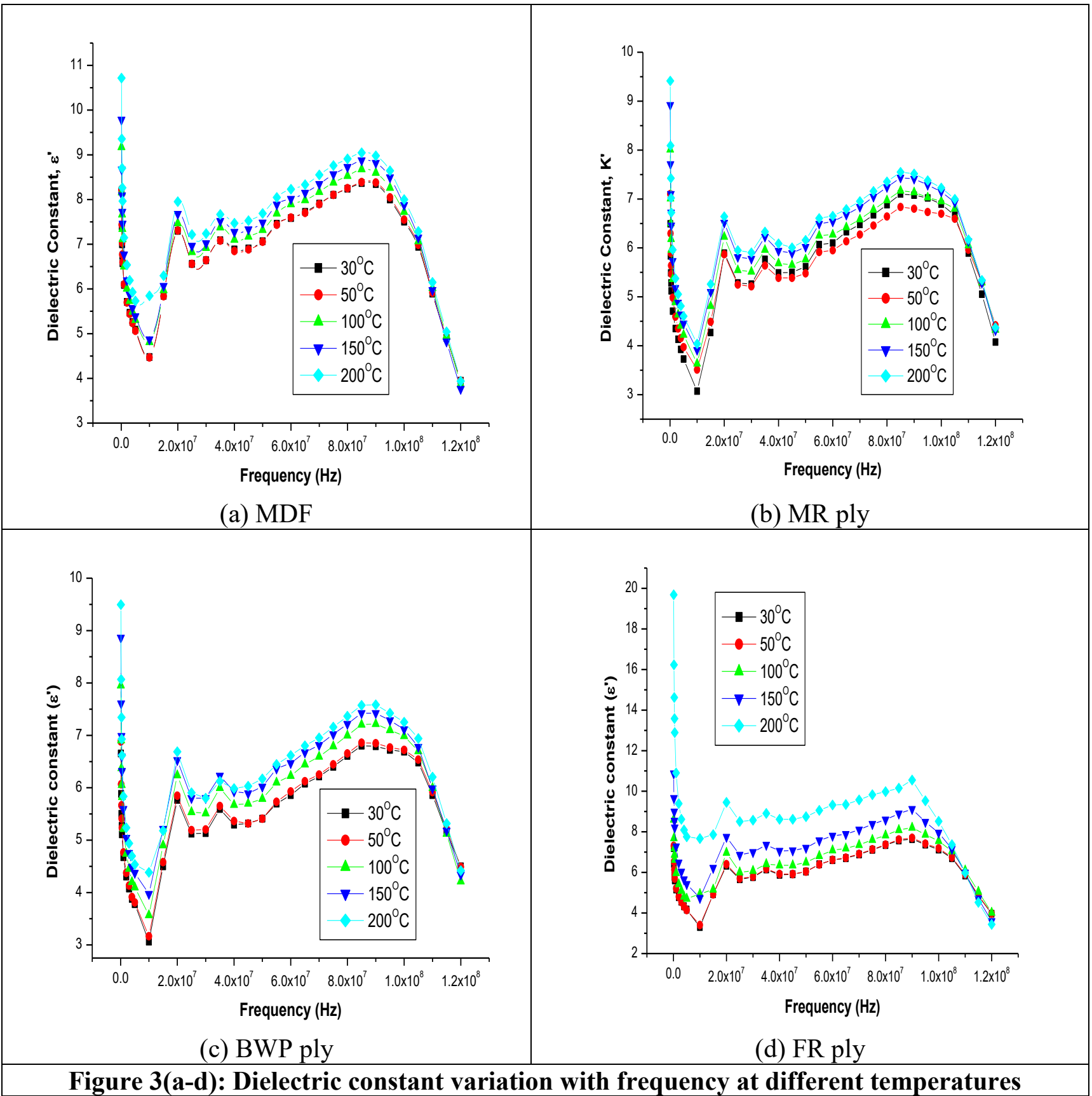




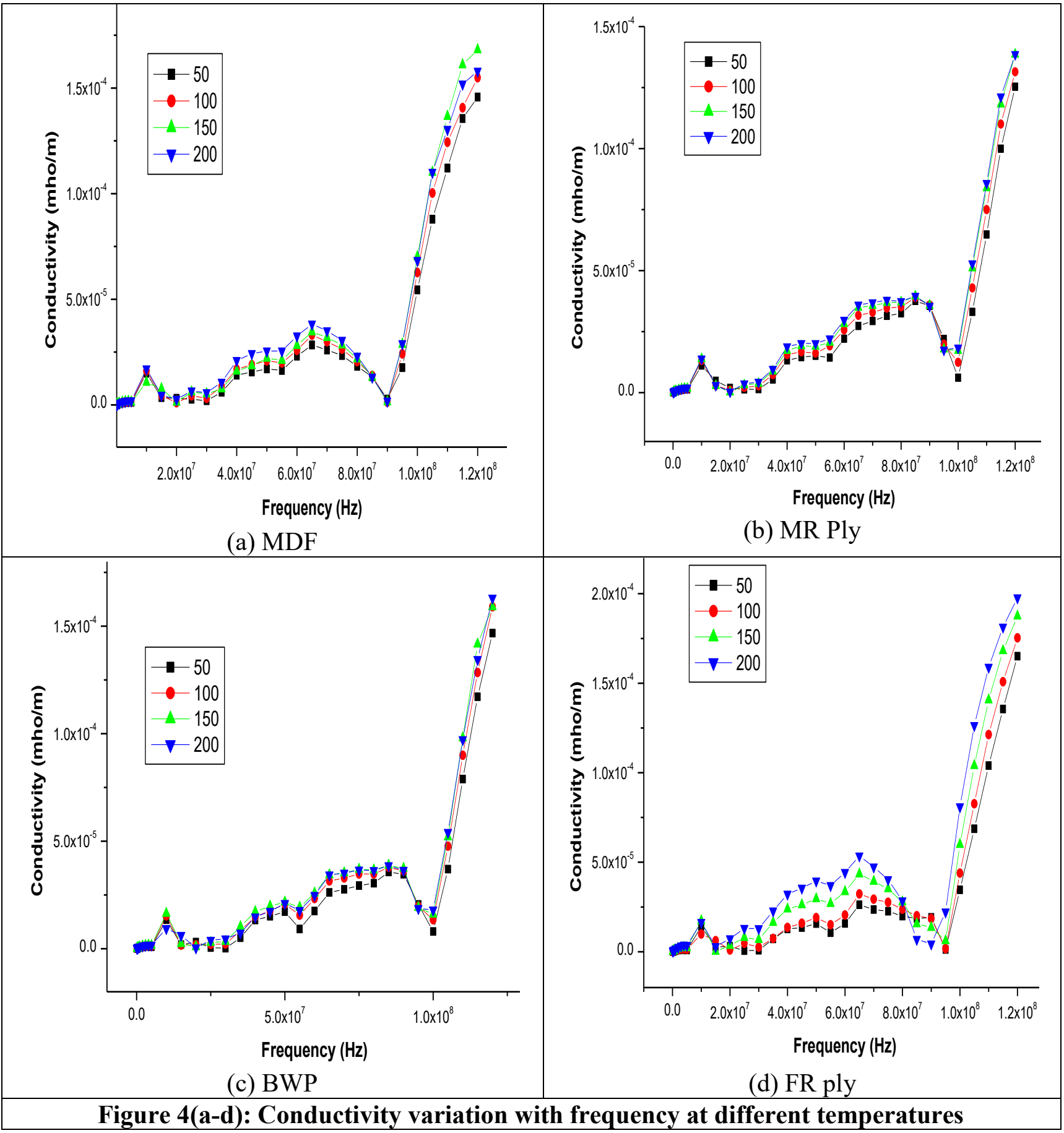




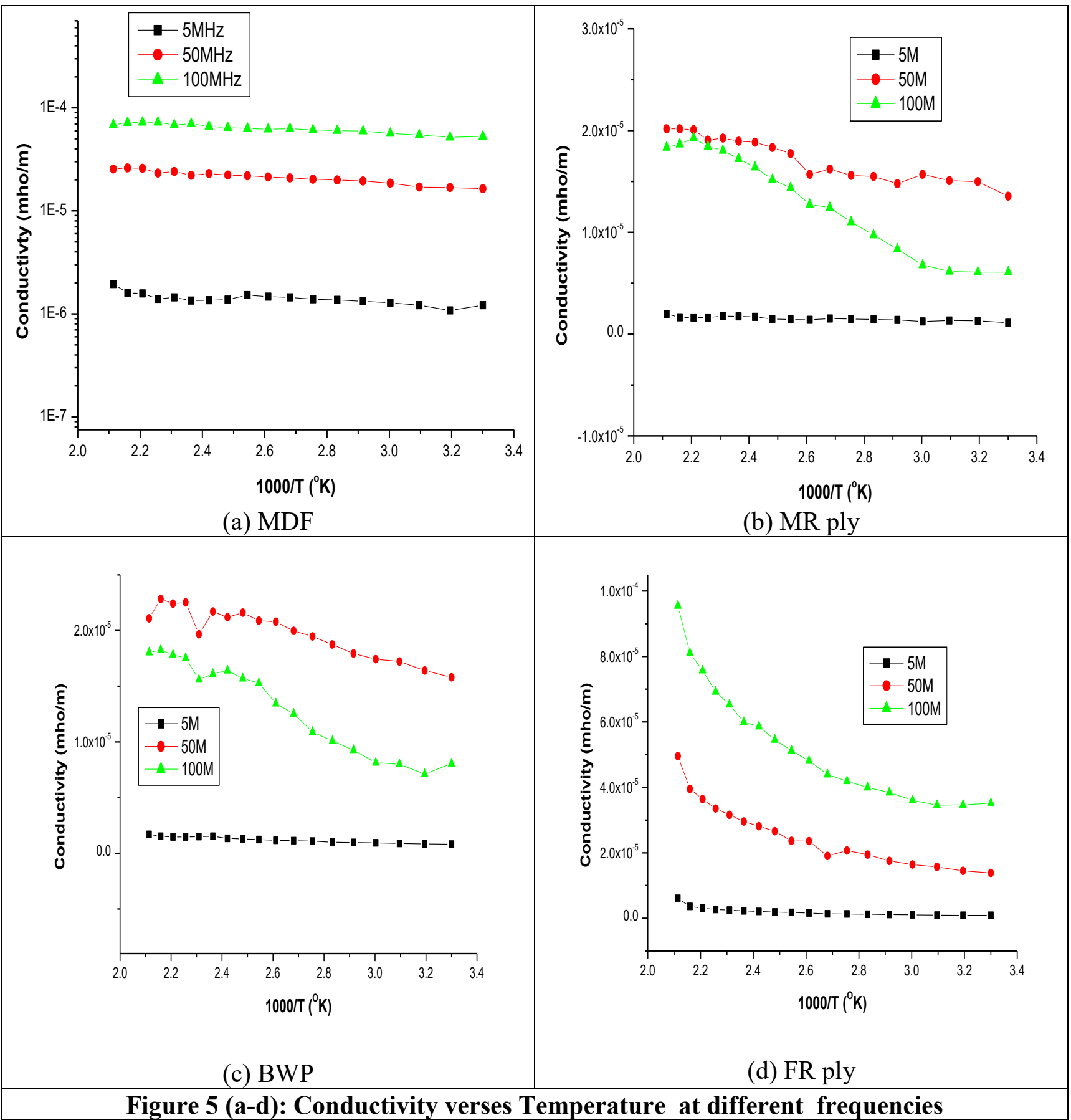

\section{REFERENCES}

[1] W.L. James, Dielectric properties of wood and hard board variation with temperature, frequency, moisture content and grain direction. No. FSRP-FPL-245. FOREST PRODUCTS LAB MADISON WIS, 1975.

[2] W.L. James, Dielectric properties of Douglas-fire at various combinations of temperature, frequency and moisture content, Forest prod. J. 27(6) (1977) 44-48.

[3] D.W. Hamil, Dielectric properties of douglas-fire measured at microwave frequencies, Forest. Prod. J. 15(2) (1965) 51-56.

[4] C. Skaar, The dielectric properties of wood at several radio- frequencies, Tech. Bull. 69 N.Y. State Coll. Forest., N.Y., 1949.

[5] J. Rafalski, Dielectric properties of compressed beech wood, Forest Prod. J. 17 (1967) 64-65.

[6] R.T. Lin, Review of the dielectric properties of wood and cellulose, Forest prod. J. 17 (1967) 61-66. 
[7] G.I. Torgovnikov, Dielectric properties of wood and wood based materials, Springer-Verlag, New York, 1993.

[8] S. Ramaswamy, B. Mohtaderi, Dielectric properties of Typical Australian wood-based biomass metrials at microwave frequency, Energy and Fuels. 24(8) (2010) 4534-4538.

[9] M Norimoto, T Yamada, The dielectric properties of wood III. The relationship between dielectric loss factor and specific gravity, J. Jan. Wood. Res. Soc. 16 (1970) 364-369.

[10]A.Z. Nanassy, Electric polarization measurement on yellow birch, Can. J. Phys. 42 (1964) 1270-1281.

[11]J. Tsutsumi, H. Watanabe, Studies on dielectric behaviour of wood. I Effect of frequency and temperature on $\varepsilon$ ' and $\varepsilon "$, Mokuzai Gakkaishi. 11(6) (1965) 232-236.

[12] Mike Golio: The RF and microwave Handbook. CRC press(2001).

[13]A.B. Lidiard, Handbuch der Physik. 20 (1957) 294.

[14]K. Funke, C. Cramer, D.Wilmer, Diffusion in condensed Matter-methods materials, models. Springer, Berlin, 2005.

[15] W. Dieterich, P. Maass, Non-Debye relaxations in disordered ionic solids, Chemical physics. 284(1) (2002) 439-467. 